\title{
Locoregionally advanced oral cavity cancer: A propensity-score matched analysis on overall survival with emphasis on the impact of adjuvant radiotherapy
}

\author{
Jacob Y. Shin MD MD $^{1}$ ～Ja Kyoung Yoon MD ${ }^{1}$ | Aaron K. Shin $\mathbf{B A}^{2}$ | \\ Aidnag Z. Diaz MD1
}

${ }^{1}$ Department of Radiation Oncology, Rush University Medical Center, Chicago, Illinois

${ }^{2}$ School of Dentistry, University of Michigan, Ann Arbor, Michigan

\section{Correspondence}

Jacob Y. Shin, Department of Radiation, Oncology Rush University Medical Center, 500 South Paulina Street, Chicago, IL 60612.

Email: jacob_shin@rush.edu

\begin{abstract}
Abstrac
Background: The purpose of this study was to determine the impact of adjuvant radiotherapy (RT) in locoregionally advanced oral cavity cancer.

Methods: Data were extracted from the National Cancer Data Base, of which overall survival (OS) is the only outcome variable available. The chi-square test and Cox regression models were used.

Results: A total of 6654 patients were identified. The utilization of adjuvant RT has increased over time. A propensity matched cohort included 3946 patients, exactly one-half of whom received adjuvant RT. Independent predictors associated with receipt of adjuvant RT included age, Charlson/Deyo comorbidity score, extracapsular extension, surgical margins, and $\mathrm{T}$ and $\mathrm{N}$ classifications. On multivariable analysis, adjuvant RT remained an independent prognosticator for OS.

Conclusion: Receipt of adjuvant RT is a prognostic factor associated with improved OS, its utilization has increased over time, and it should be considered for clinically suitable patients who have undergone resection for the disease.
\end{abstract}

\section{K E Y W O R D S}

adjuvant radiotherapy, locoregionally advanced, oral cavity cancer, outcomes, prognostic factors

\section{1 | INTRODUCTION}

In 2017, Siegel et $\mathrm{al}^{1}$ estimated that there would be 32670 new cases of oral cavity cancer in the United States and an estimated 6650 deaths. Factors affecting prognosis in those with oral cavity cancer include nodal involvement, extracapsular extension, surgical margins, tumor size, perineural invasion, and tumor classification. ${ }^{2}$ In those with locoregionally advanced oral cavity cancers, up-front resection is recommended for those patients who present with resectable disease. $^{3}$ These patients, however, are at significant risk for locoregional recurrence after resection and are generally recommended for adjuvant therapy. ${ }^{4-9}$ Per the National Comprehensive Cancer Network guidelines, postoperative adjuvant radiotherapy (RT) is the recommended treatment for those presenting with risk features, including pT3 or pT4 primary, $\mathrm{N} 2$ or N3 nodal disease, or perineural invasion, and adjuvant systemic therapy/RT is recommended for those with extracapsular nodal extension or positive surgical margins. ${ }^{3}$

Limited data exist regarding the specific impact of adjuvant $\mathrm{RT}$ in those with locoregionally advanced disease particularly focused on primary tumors arising from the oral cavity. The purpose of this hospital-based retrospective study on 6654 patients diagnosed with squamous cell carcinoma of the oral cavity was to determine the prognostic factors for overall survival (OS) and the utilization and impact of adjuvant RT in those who have undergone resection for the disease. 


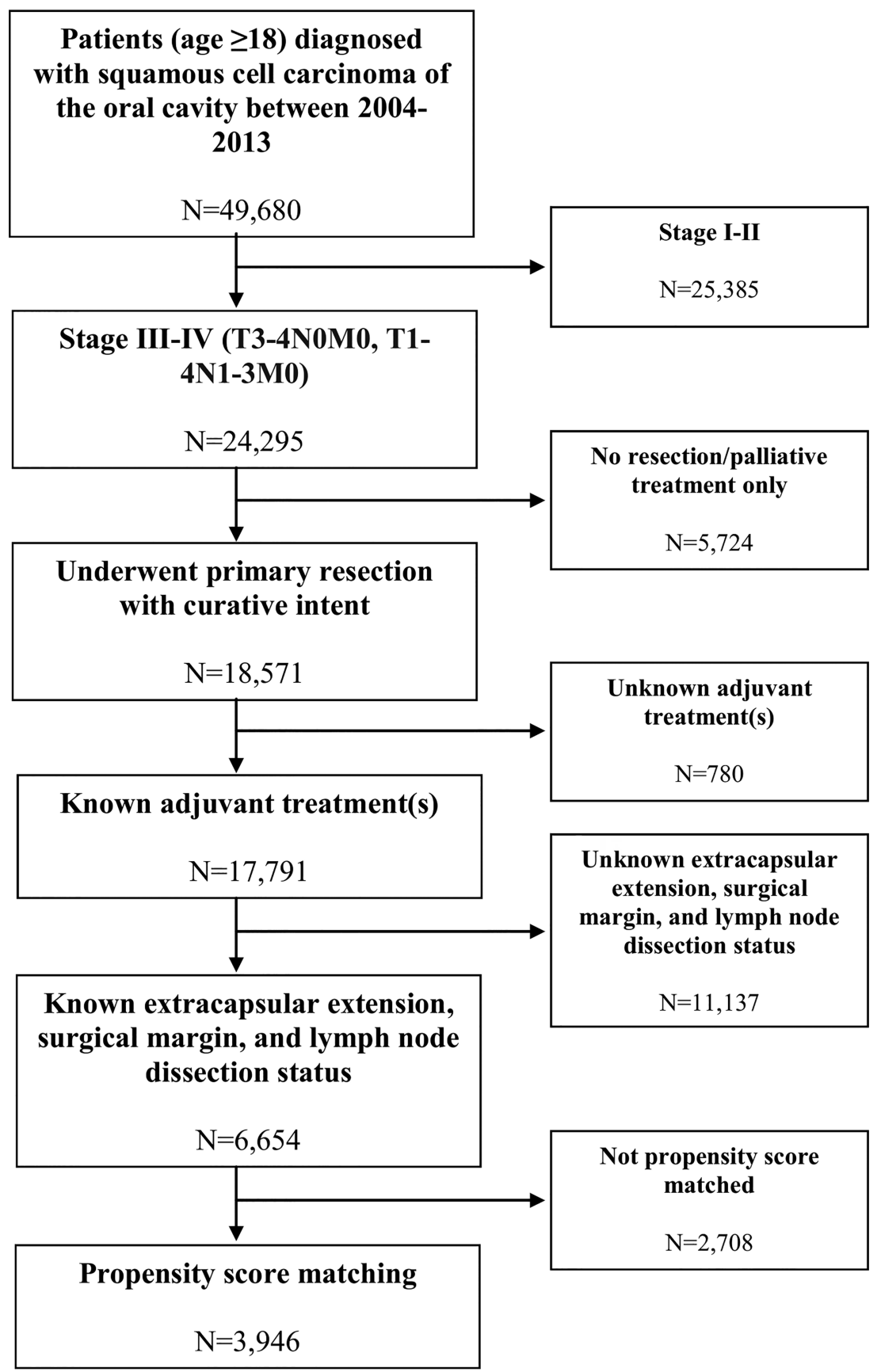

FIGURE 1 Flowchart describing the composition of the patient cohort

\section{2 | MATERIALS AND METHODS}

\section{1 | Patient population}

Data were extracted from the National Cancer Data Base (NCDB), which include hospital registry data collected from $>1500$ Commission on Cancer-accredited facilities representing $70 \%$ of newly diagnosed cancer cases annually in the United States. ${ }^{10}$ It is a joint project of the Commission on Cancer of the American College of Surgeons and the American Cancer Society. The data used in our investigation are derived from a deidentified NCDB file. The American
College of Surgeons and the Commission on Cancer have not verified and are not responsible for the analytic or statistical methodology used, or the conclusions drawn from these data by the investigators. The only outcome variable available in the NCDB is OS. This study was approved by the institutional review board of Rush University Medical Center, Chicago, IL, which waived the need for informed consent for use of these deidentified data.

As seen in Figure 1, our study population consists of 6654 patients diagnosed with locoregionally advanced squamous cell carcinoma of the oral cavity, including the anterior two-thirds of the tongue, lip, gingiva, floor of mouth, hard 
palate, buccal mucosa, vestibule of mouth, retromolar trigone, and oral cavity not otherwise specified (NOS; International Classification of Disease-0-3 histology code 8070/38078/3) from 2004 to 2013. Classification groups III to IV, including those with T3-4N0M0 and T1-4N1-3M0 disease as defined by the American Joint Committee on Cancer 2017 classification system, were considered locoregionally advanced. ${ }^{11}$ All study patients were 18 years or older and underwent excision of the primary tumor with curative intent. Patients receiving adjuvant RT received external beam RT. Additional exclusion criteria included patients with no record regarding $\mathrm{T}, \mathrm{N}$, or M classifications, extracapsular extension or surgical margin status, or receipt of adjuvant RT or chemotherapy.

Comorbidities, as described by the Charlson/Deyo comorbidity score, were defined by a weighted score derived from the sum of the scores for each of the comorbid conditions listed in the Charlson Comorbidity Score Mapping Table. ${ }^{12,13}$ A score of 0 indicated no significant comorbid conditions and higher scores indicated greater comorbidity burden. Because of the small proportion of patients with a Charlson Comorbidity score exceeding 2, the NCDB has truncated the data to 0,1 , and $2(>1)$. Insurance status was identified as the patient's primary insurance carrier at the time of initial diagnosis and/or treatment. Categories, as supplied by the NCDB for insurance status, include private, uninsured, Medicaid, Medicare, and other governmental.

\section{2 | Statistical analysis}

All data analyses were performed using SPSS 23.0 (IBM, Armonk, NY). Proportional distribution of demographic and clinicopathologic factors, and treatment by receipt of adjuvant RT were compared using the 2-tailed chi-square test. The chi-square test of independence using Fisher's exact test determined whether there was an association between categorical variables. A cross-tabulation was performed, and the $P$ value (significance level of .05) was used to determine whether the null hypothesis (no significant difference between specified populations; any observed difference being due to sampling error) could be rejected and whether a statistical association between categorical variables existed. The primary study end point was OS, which was defined as time to death from the date of diagnosis of squamous cell carcinoma of the oral cavity. Participating Commission on Cancer-accredited registries report patient follow-ups to the NCDB annually. The NCDB records the number of months between the date of diagnosis and the date on which the patient was last contacted or died. The NCDB does not include cause of death information, so cause-specific survival cannot be calculated. Factors significant on univariate OS analysis were included in Cox regression multivariable analysis, which was used to compute hazard ratios (HRs) with
95\% confidence intervals (CIs) to identify independent prognostic factors for OS using a forward selection variable selection process. To adjust further for the effect of potential confounding variables, a stepwise binary logistic regression was used to determine predictors of adjuvant RT use in our patient population. A propensity score model for the likelihood of receiving adjuvant RT was developed, including the covariates age at diagnosis, sex, race, Charlson/Deyo comorbidity score, insurance status, site of disease, extracapsular extension status, surgical margin status, $\mathrm{T}$ classification, $\mathrm{N}$ classification, tumor grade, and receipt of adjuvant chemotherapy. One-to-one propensity matching was then performed with the fixed-caliper width set to 0.01 , which has demonstrated negligible relative bias (relative bias ranging from $-2 \%$ to $3 \%$ ) and greater precision for estimating treatment effects. ${ }^{14}$ These 2 cohorts (ie, adjuvant RT vs no adjuvant RT) were compared using the log-rank test, and the HR for OS was calculated using Cox regression. A landmark analysis was also performed establishing a landmark time, including only those patients who survived to this time point and followed forward in time to evaluate whether OS is associated with receipt of adjuvant RT. The 3-month landmark was chosen a priori because this time point corresponds to the usual interval at which a reclassification evaluation to assess the effect of treatment would occur. ${ }^{3}$ Kaplan-Meier methods were used to estimate survival probabilities. A 2sided $P$ value $<.05$ was considered statistically significant.

\section{3 | RESULTS}

\section{1 | Patient and treatment characteristics}

A total of 6654 patients diagnosed with locoregionally advanced squamous cell carcinoma of the oral cavity who underwent primary tumor resection in 2004-2013 were identified (Table 1). The median age was 63 years (range 18-90 years). The population was predominately male, who were white, had a Charlson/Deyo comorbidity score of 0 , and carried private insurance or Medicare. A total of 4287 patients (64.4\%) received adjuvant RT and 2367 (35.6\%) did not.

Independent predictors associated with receipt of adjuvant RT are shown in Table 2. These included younger age, lower Charlson/Deyo comorbidity score, extracapsular extension, positive surgical margins, and $\mathrm{T}$ and $\mathrm{N}$ classifications of disease. Over the study time period 2004-2013, the utilization of adjuvant RT significantly increased from $43.1 \%$ in 2004 to $64.1 \%$ in 2013 ( $P=.019$, chi-square test; Figure 2$)$.

\section{2 | Overall survival}

The median time to follow-up was 22.2 months (range 1-126 months). Univariate analysis demonstrated that those who received adjuvant RT (HR 0.756, 95\% CI 0.692-0.825) were 
TABLE 1 Patient characteristics stratified by receipt of adjuvant radiotherapy

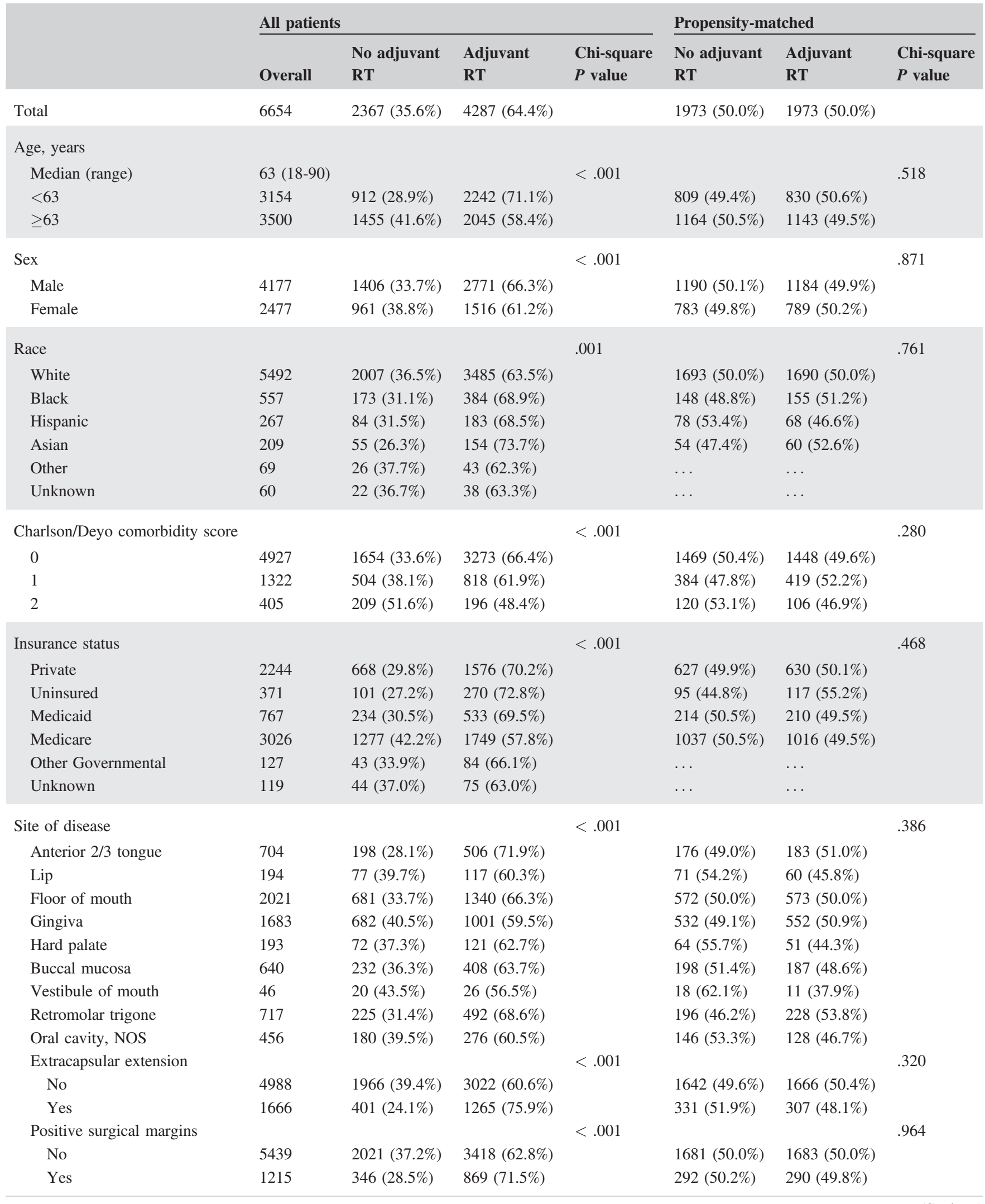


TABLE 1 (Continued)

\begin{tabular}{|c|c|c|c|c|c|c|c|}
\hline & \multicolumn{4}{|c|}{ All patients } & \multicolumn{3}{|c|}{ Propensity-matched } \\
\hline $\mathrm{T}$ classification & & & & $<.001$ & & & .209 \\
\hline 1 & 619 & $216(34.9 \%)$ & $403(65.1 \%)$ & & $188(53.6 \%)$ & $163(46.4 \%)$ & \\
\hline 2 & 705 & $193(27.4 \%)$ & $512(72.6 \%)$ & & $178(48.5 \%)$ & $189(51.5 \%)$ & \\
\hline $\mathrm{N}$ classification & & & & $<.001$ & & & .171 \\
\hline 0 & 2814 & $1266(45.0 \%)$ & $1548(55.0 \%)$ & & $1002(49.1 \%)$ & $1037(50.9 \%)$ & \\
\hline 1 & 1343 & $492(36.6 \%)$ & $851(63.4 \%)$ & & $428(50.5 \%)$ & $419(49.5 \%)$ & \\
\hline 2 & 2450 & $596(24.3 \%)$ & $1854(75.7 \%)$ & & $531(50.9 \%)$ & $513(49.1 \%)$ & \\
\hline 1 & 1079 & $440(40.8 \%)$ & $639(59.2 \%)$ & & $359(51.1 \%)$ & $344(48.9 \%)$ & \\
\hline 2 & 4036 & $1430(35.4 \%)$ & $2606(64.6 \%)$ & & $1196(50.4 \%)$ & $1177(49.6 \%)$ & \\
\hline 3 & 1273 & $398(31.3 \%)$ & $875(68.7 \%)$ & & $338(47.1 \%)$ & $380(52.9 \%)$ & \\
\hline Unknown & 266 & $99(37.2 \%)$ & $167(62.8 \%)$ & & $80(52.6 \%)$ & $72(47.4 \%)$ & \\
\hline Adjuvant chemotherapy & & & & $<.001$ & & & 0.999 \\
\hline No & 4405 & $2264(51.4 \%)$ & $2141(48.6 \%)$ & & $1877(50.0 \%)$ & $1877(50.0 \%)$ & \\
\hline Yes & 2249 & $103(4.6 \%)$ & $2146(95.4 \%)$ & & $96(50.0 \%)$ & $96(50.0 \%)$ & \\
\hline
\end{tabular}

Abbreviations: NOS, not otherwise specified; RT, radiotherapy.

associated with significantly better OS than those who did not (Table 3). Additionally, age at diagnosis, Charlson/Deyo comorbidity score, insurance status, extracapsular extension, surgical margins, $\mathrm{T}$ and $\mathrm{N}$ classifications of disease, and tumor grade were statistically significant outcome factors. On Cox regression multivariable analysis, receipt of adjuvant RT (HR 0.640, 95\% CI 0.582-0.704) remained an independent prognosticator for improved OS compared to nonreceipt (Table 3). Additionally, older age at diagnosis $(P<.001)$, higher Charlson/Deyo comorbidity score (0 vs 2: HR 1.646 , $P<.001$ ), extracapsular extension (HR 1.469, $P<.001$ ), positive surgical margins (HR 1.386, $P<.001$ ), higher T classification ( 1 vs 2 : HR $1.504, P<.001 ; 1$ vs 3 : HR $2.101, P<.001 ; 1$ vs 4 : HR 2.160, $P<.001$ ), and higher $\mathrm{N}$ classification of disease ( 0 vs 1 : HR $1.531, P<.001 ; 0$ vs 2 : HR 2.318, $P<.001 ; 0$ vs 3: HR 2.662, $P<.001$ ) were significant prognostic factors associated with worse OS.

\section{3 | Sensitivity analyses}

The propensity model was created, and the resultant matched cohort included 3946 patients, exactly one half of whom received adjuvant RT. There was an expected balance of covariates between the 2 groups (Table 1). The HR from a univariate Cox regression was $0.683(P<.001)$, and from a multivariable Cox regression it was $0.642(P<.001$; Table
4). Landmark analysis was used to evaluate the association of adjuvant RT to OS. A 3-month landmark was chosen. For patients diagnosed from 2004-2013 who survived at least 3 months, the median survivals for patients treated with and without adjuvant RT were 51.3 months and 42.7 months, respectively, and the 5-year OS probabilities were $47.9 \%$ versus $39.4 \%(P<.001$; Figure 3$)$.

\section{4 | Subset analysis}

In terms of patient characteristics, a statistically significant 5year OS benefit was observed in those $\geq 63$ years $(43.6 \%$ vs $31.9 \%, P<.001)$, whereas there was a trend toward significance in those $<63$ years $(53.5 \%$ vs $44.4 \%, P=.076$; Table 5). Those with a Charlson/Deyo comorbidity score of 0 or 1 had an associated 5-year OS benefit with receipt of adjuvant RT (0: $47.8 \%$ vs $38.0 \%, P<.001 ; 1: 54.6 \%$ vs $33.5 \%, P<$ $.001)$, whereas those with a Charlson/Deyo comorbidity score of 2 did not have a demonstrated OS benefit $(P=$ .635). A 5-year OS benefit was associated to adjuvant RT regardless of extracapsular extension status (no: $50.5 \%$ vs $41.4 \%, P<.001$; yes: $32.3 \%$ vs $15.0 \%, P<.001)$ or surgical margin status (negative: $49.0 \%$ vs $40.0 \%, P<.001$; positive: $39.9 \%$ vs $19.8 \%, P<.001)$. In those with no extracapsular extension and negative surgical margins, no derived OS benefit with adjuvant RT was observed in those with $\mathrm{N} 2$ disease 
TABLE 2 Independent predictors associated with adjuvant radiotherapy

\begin{tabular}{|llll}
\hline Variables & HR & $\mathbf{9 5 \%}$ CI & $\boldsymbol{P}$ value \\
\hline $\begin{array}{l}\text { Age, years } \\
<63\end{array}$ & Reference & & \\
$\geq 63$ & 0.721 & $0.623-0.834$ & $<.001$ \\
\hline Sex & & & \\
Man & Reference & & \\
Woman & 0.895 & $0.800-1.002$ & .053 \\
\hline Race & & & \\
Black & & & \\
White & Reference & & \\
Hispanic & 0.866 & $0.706-1.062$ & .166 \\
Asian & 1.036 & $0.743-1.445$ & .835 \\
\hline Charlson/Deyo comorbidity score & & .221 \\
0 & 1.267 & $0.868-1.849$ & \\
1 & Reference & & \\
2 & 0.844 & $0.738-0.965$ & .013 \\
\hline
\end{tabular}

Insurance status

$\begin{array}{llll}\text { Private } & \text { Reference } & & \\ \text { Uninsured } & 1.016 & 0.785-1.314 & .905 \\ \text { Medicaid } & 0.832 & 0.689-1.006 & .057 \\ \text { Medicare } & 0.762 & 0.657-0.885 & <.001\end{array}$

Site

Anterior $2 / 3$ tongue

Lip

Reference

Floor of mouth

Gingiva

0.839

0.819

Hard palate

0.745

Buccal mucosa

0.797

Vestibule of mouth

0.577

1.010

Retromolar trigone

0.713

Extracapsular extension No

Yes

1.303

$1.112-1.527$

.001

Positive surgical margins

No

Reference

Yes

1.388

1.199-1.607

$<.001$

\section{$\mathrm{T}$ classification}

$\begin{array}{llll}1 & \text { Reference } & & \\ 2 & 1.295 & 1.012-1.658 & .040 \\ 3 & 1.065 & 0.804-1.411 & .661 \\ 4 & 1.254 & 1.021-1.540 & .031 \\ & & & \\ \text { N classification } & & & \\ 0 & \text { Reference } & & \\ 1 & 1.299 & 1.104-1.528 & .002 \\ 2 & 2.004 & 1.710-2.349 & <.001 \\ 3 & 1.474 & 0.739-2.941 & .271 \\ & & & \text { (Continues) }\end{array}$

TABLE 2 (Continued)

\begin{tabular}{llll} 
Variables & HR & $\mathbf{9 5 \%}$ CI & $\boldsymbol{P}$ value \\
Grade & & & \\
1 & Reference & & \\
2 & 1.043 & $0.899-1.210$ & .581 \\
3 & 1.136 & $0.944-1.366$ & .177 \\
Unknown & 0.956 & $0.708-1.290$ & .767 \\
\hline
\end{tabular}

Abbreviations: CI, confidence interval; HR, hazard ratio.

(30.9\% vs $29.5 \%, P=.073)$. Those with higher T classifications were associated to a derived 5-year OS benefit with adjuvant RT (T3: $43.6 \%$ vs $24.2 \%, P=.003$; T4: $47.8 \%$ vs $38.2 \%, P<.001)$.

\section{4 | DISCUSSION}

We investigated the impact of adjuvant RT on OS in patients with locoregionally advanced squamous cell carcinoma of the oral cavity, and our study demonstrated important findings. First, the utilization of adjuvant RT for these patients with advanced disease has significantly increased over the past decade. Next, those younger, or with less comorbidity burden, extracapsular extension of disease, and positive surgical margins, and higher $\mathrm{T}$ and $\mathrm{N}$ classifications of disease are more likely to receive adjuvant RT. Conversely, those who were older, with higher comorbidity burden, and with less adverse features seen on final pathology status postresection are less likely to receive adjuvant RT. Finally, our data suggest that adjuvant RT is significantly associated with improved OS, which was maintained on multivariable and landmark analyses among propensity-matched patients.

For those with locoregionally advanced squamous cell carcinoma of the oral cavity, the National Comprehensive Cancer Network recommends surgery and neck dissection for resectable disease. ${ }^{3}$ Given the extent of disease, these patients are at significant risk for local recurrence after resection. Unfavorable prognostic factors for local control in the postoperative setting include close or positive surgical margins, extracapsular extension, tumor depth, perineural invasion, and nodal metastasis, and patients with combinations of these adverse pathologic features have been associated with significantly decreased OS in addition to decreased locoregional control rates. ${ }^{15,16}$ A 35-year single institutional study on 226 patients with primary oral cavity cancer treated with adjuvant RT found that these factors were significant prognosticators for locoregional control on multivariable analysis. ${ }^{17}$

Positive surgical margins and extracapsular extension in particular are 2 unfavorable high-risk factors, ${ }^{18-20}$ and our subset analyses, including propensity-matched patients, suggest that a significant absolute benefit for adjuvant RT was 


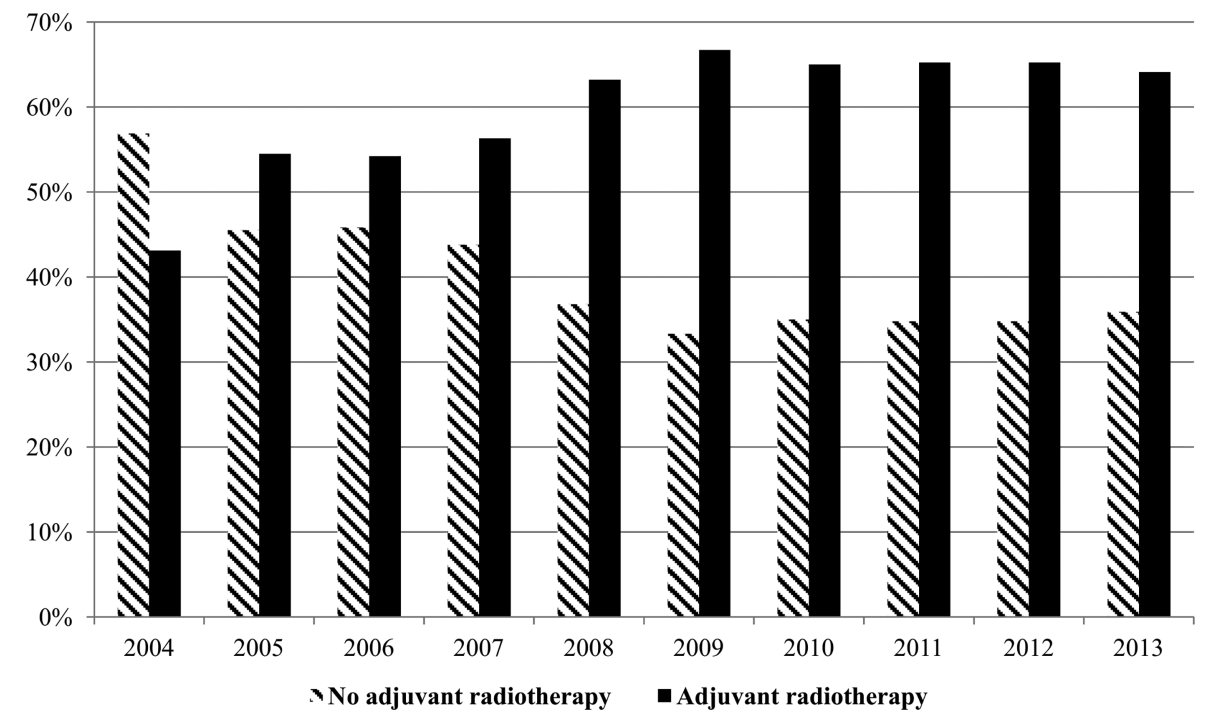

F IG URE 2 Utilization of adjuvant radiotherapy over the time period 2004-2013 ( $P=.019$, chi-square test)

TABLE 3 Cox regression univariate and multivariable analysis for overall survival for all patients

\begin{tabular}{|c|c|c|c|c|c|c|}
\hline \multirow[b]{2}{*}{ Variables } & \multicolumn{3}{|c|}{ Univariate } & \multicolumn{3}{|c|}{ Multivariable } \\
\hline & HR & 95\% CI & $P$ value & HR & 95\% CI & $P$ value \\
\hline Age at diagnosis ${ }^{\mathrm{a}}$ & 1.021 & $1.017-1.025$ & $<.001$ & 1.021 & $1.016-1.026$ & $<.001$ \\
\hline \multicolumn{7}{|l|}{ Sex } \\
\hline Male & Reference & & & ND & & \\
\hline \multicolumn{7}{|l|}{ Race } \\
\hline Black & Reference & & & ND & & \\
\hline White & 0.973 & $0.836-1.132$ & .973 & & & \\
\hline Hispanic & 0.794 & $0.605-1.043$ & .794 & & & \\
\hline Asian & 0.832 & $0.611-1.131$ & .832 & & & \\
\hline
\end{tabular}

Charlson/Deyo comorbidity score

$$
\begin{aligned}
& 0 \\
& 1
\end{aligned}
$$$$
2
$$

Reference

1.182

1.870
1.063-1.314

1.602-2.184

\section{Reference}

1.094

1.419

1.650

Medicaid

Medicare

Private

Uninsured
Site

Anterior 2/3 tongue

Lip

Floor of mouth

Gingiva

Hard palate

Buccal mucosa

Vestibule of mouth

Retromolar trigone
Reference

0.704

1.006

0.861

1.275

1.189

1.268

0.967

$\begin{array}{ll}0.887-1.350 & .401 \\ 1.225-1.644 & <.001 \\ 1.492-1.824 & <.001\end{array}$

$1.492-1.824$
Reference

$.002 \quad 1.094$

$<.001 \quad 1.646$

Reference

$\begin{array}{lll}1.104 & 0.893-1.364 & .361 \\ 1.429 & 1.230-1.659 & <.001 \\ 1.263 & 1.118-1.427 & <.001\end{array}$

0.982-1.219

1.404-1.930
.102

$<.001$ 
TABLE 3 (Continued)

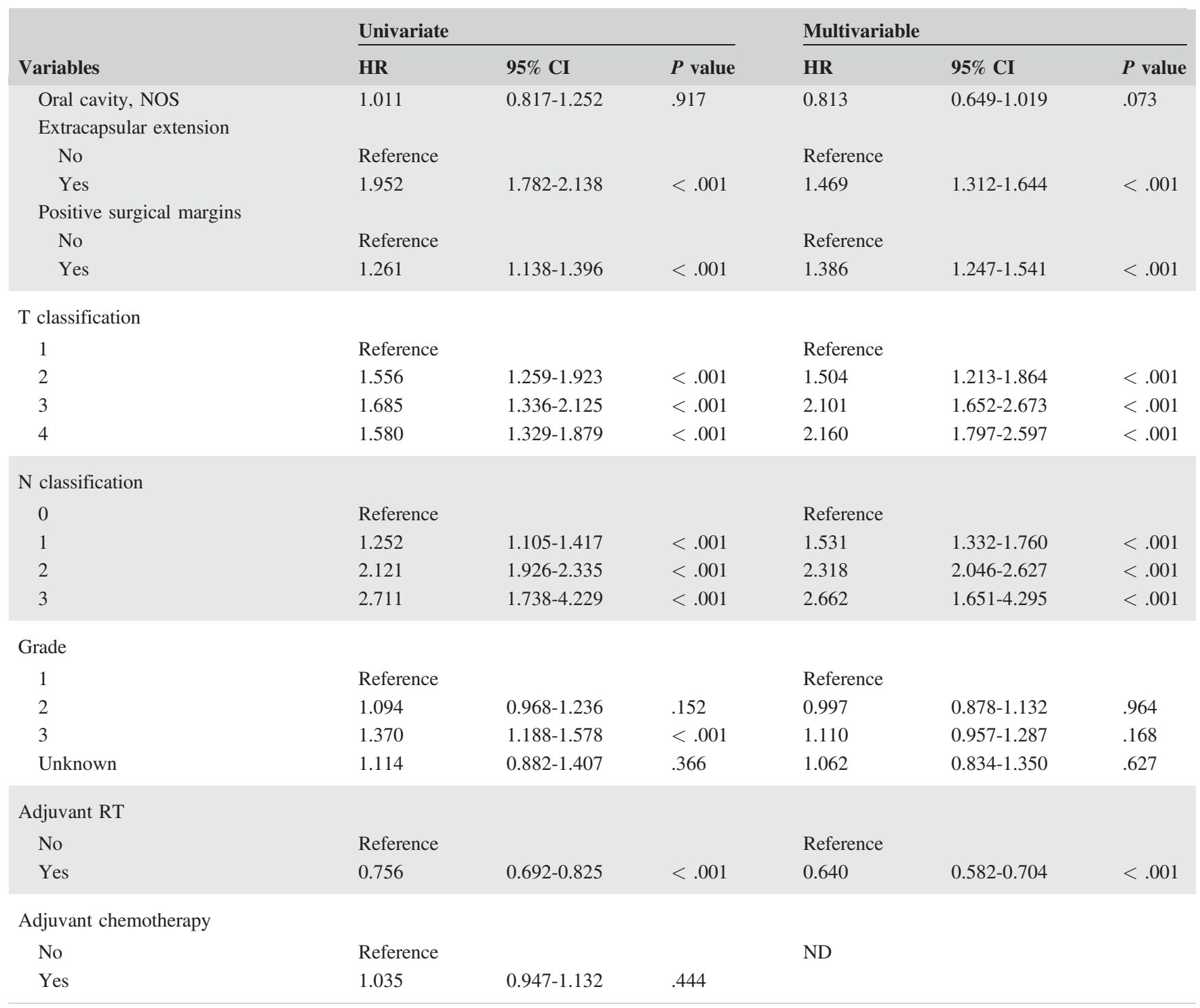

Abbreviations: CI, confidence interval; HR, hazard ratio; ND, not determined; NOS, not otherwise specified; RT, radiotherapy.

${ }^{\mathrm{a}}$ Analyzed as a continuous variable.

derived in patients with either of these high-risk features. The presence of $\mathrm{T} 3$ to $\mathrm{T} 4$ disease without involved margins or extracapsular extension was also associated with a significant OS benefit when adjuvant RT was received. These results strongly argue for the importance of adjuvant RT for patients with adverse pathologic features. A phase III randomized trial conducted in India on 900 patients with locally advanced and resectable squamous cell carcinoma of the oral cavity found 5-year locoregional control to be $58.2 \%-65.1 \%$ for those receiving adjuvant RT or chemoradiotherapy, ${ }^{4}$ of whom $90 \%$ had T3 to T4 tumors, and $48 \%$ had N2 to N3 disease. Very few patients had positive margins; although $54.7 \%$ had extracapsular extension and 329 (36.5\%) had multiple nodal involvements. Additionally, our data demonstrated that receipt of adjuvant RT in patients with locoregionally advanced disease with moderately or poorly differentiated tumors are also associated with an OS benefit, suggesting that tumor grade should be given consideration as an adverse risk feature to be used on multivariable models in this cohort. This result is consistent with a Surveillance, Epidemiology, and End Results study published by Thomas et $\mathrm{al}^{21}{ }^{21}$ which demonstrated a strong association between tumor grade and disease-specific survival for patients with classifications I to II with squamous cell carcinoma of the oral cavity and noted an adjusted risk of death 2.7 times greater if the tumor was poorly differentiated or undifferentiated compared to well-differentiated tumors.

In contrast, there may be those who may not derive an OS benefit with the addition of adjuvant RT. In a randomized trial on patients with advanced head and neck squamous cell carcinoma, 213 patients were prospectively studied with $15 \%$ having no adverse pathologic factors and receiving no 
TABLE 4 Cox regression univariate and multivariable analysis for overall survival for propensity-matched patients

\begin{tabular}{|c|c|c|c|c|c|c|}
\hline Variable & \multicolumn{3}{|c|}{ Univariate } & \multicolumn{3}{|c|}{ Multivariable } \\
\hline Age at diagnosis ${ }^{\mathrm{a}}$ & 1.025 & $1.020-1.030$ & $<.001$ & 1.024 & $1.018-1.031$ & $<.001$ \\
\hline \multicolumn{7}{|l|}{ Sex } \\
\hline Male & Reference & & & \multicolumn{3}{|l|}{ ND } \\
\hline \multicolumn{7}{|l|}{ Race } \\
\hline Black & Reference & & & & & \\
\hline White & 0.921 & $0.752-1.128$ & .427 & 0.914 & $0.741-1.127$ & .400 \\
\hline Hispanic & 0.656 & $0.445-0.968$ & .033 & 0.665 & $0.449-0.984$ & .042 \\
\hline Asian & 0.797 & $0.530-1.197$ & .274 & 0.896 & $0.591-1.356$ & .602 \\
\hline 2 & 1.964 & $1.603-2.406$ & $<.001$ & 1.776 & $1.447-2.181$ & $<.001$ \\
\hline \multicolumn{7}{|l|}{ Insurance status } \\
\hline Private & Reference & & & Refere & & \\
\hline Uninsured & 1.170 & $0.875-1.564$ & .290 & 1.192 & $0.889-1.600$ & .241 \\
\hline Medicaid & 1.603 & $1.309-1.963$ & $<.001$ & 1.560 & $1.265-1.925$ & $<.001$ \\
\hline Medicare & 1.828 & $1.597-2.093$ & $<.001$ & 1.318 & $1.120-1.550$ & .001 \\
\hline \multicolumn{7}{|l|}{ Site } \\
\hline Anterior $2 / 3$ tongue & Reference & & & Refere & & \\
\hline Oral cavity, NOS & 0.990 & $0.739-1.325$ & .944 & 0.885 & $0.654-1.197$ & .427 \\
\hline
\end{tabular}

Extracapsular extension

No

Yes

Positive surgical margins

No

Yes

$\mathrm{T}$ classification

1

2

3

4
Reference

2.703

Reference

1.654

1.438-1.903

$<.001$

Reference

1.494

1.590

1.304
1.131-1.972

1.183-2.139

1.043-1.630
.005

.002

.020

Reference

1.745

1.490-2.044

$<.001$

Reference

$1.425 \quad 1.236-1.644<.001$

$\mathrm{N}$
0
1
2
3
Reference

1.409

2.785

3.947
1.210-1.639

2.453-3.162

$1.764-8.830$
$<.001$

$<.001$

.001
Reference

$\begin{array}{lll}1.356 & 1.026-1.792 & .033 \\ 2.018 & 1.480-2.750 & <.001 \\ 1.924 & 1.514-2.444 & <.001\end{array}$

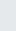

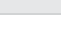

(1)

Reference

1.578
2.341
2.481

$\begin{array}{ll}1.329-1.873 & <.001 \\ 1.997-2.745 & <.001 \\ 1.091-5.645 & .030\end{array}$


TABLE 4 (Continued)

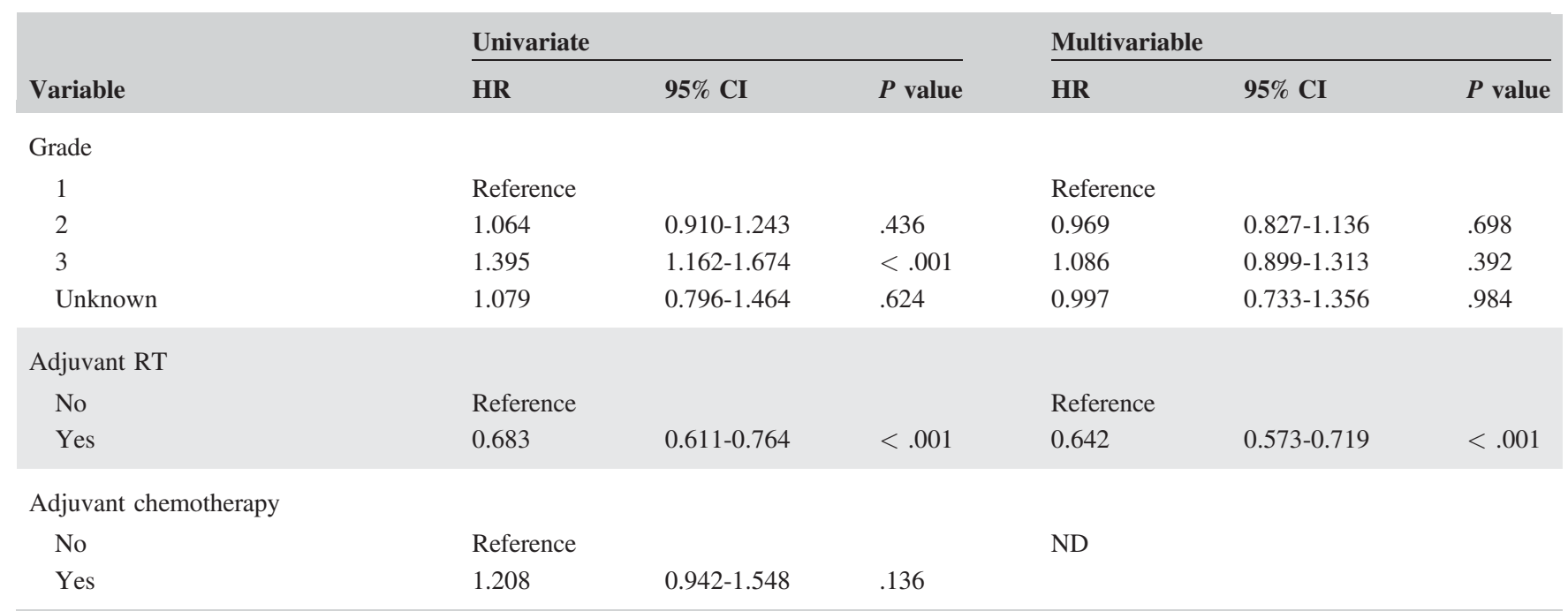

Abbreviations: CI, confidence interval; HR, hazard ratio; ND, not determined; NOS, not otherwise specified; RT, radiotherapy.

analyzed as a continuous variable.

adjuvant therapy, and had a 5-year locoregional control rate of $83 \% .^{22}$ In the absence of extracapsular extension or positive surgical margins, our data demonstrated that adjuvant RT was not associated with an OS benefit in patients with $\mathrm{N} 2$ disease who underwent primary resection and lymph node dissection in our propensity-matched analysis. Additionally, those with less aggressive disease, including those with $\mathrm{T} 1$ to $\mathrm{T} 2$ and grade 1 disease, respectively, were not associated with an OS benefit upon receipt of adjuvant RT. These findings suggest that certain patient subsets with locoregionally advanced disease undergoing primary resection with lymph node dissection and ultimately more favorable final pathologic findings have a good prognosis with surgery alone. In terms of patient characteristics, no statistically significant OS benefit associated with adjuvant RT was observed in those with a Charlson/Deyo comorbidity score of 2. Reasons for this are likely multifactorial in etiology but previous studies related to the prognostic impact of comorbidity and cancer have found that although comorbidity does not seem to be associated with more aggressive types of cancer or other differences in tumor biology, postoperative complications, and mortality are higher and the chance of completing a course of cancer treatment is lower for those with great comorbidity burden. ${ }^{23}$

Our data suggest that the use of adjuvant RT has grown over the past decade, and reasons for this can only be hypothesized given the limitations of the NCDB but are also likely multifactorial in etiology. The use of intensitymodulated radiotherapy for head and neck cancer, which offers improvements in side effect profile and quality of life when compared with conventional RT techniques, ${ }^{24}$ has significantly increased with $1.3 \%$ of patients receiving it in 2000 compared to $46.1 \%$ in $2005(P<.001)$ as demonstrated in a Surveillance, Epidemiology, and End Results-Medicare analysis on 5487 patients, although significant geographic variation was noted. ${ }^{25}$ We did not report intensity-modulated RT use as a variable in our study given that the NCDB and its radiation technique options(eg, conventional vs 3D conformal vs intensity-modulated RT) are not mutually exclusive. Insurance status has been associated with disparities in treatment delivery for head and neck cancers with those patients who are insured more likely to receive definitive treatment than those who are uninsured. ${ }^{26}$ The Affordable Care Act was introduced in 2010 and launched major regulatory changes of the U.S. healthcare system with the goal to increase patient access to health insurance coverage. ${ }^{27}$ In

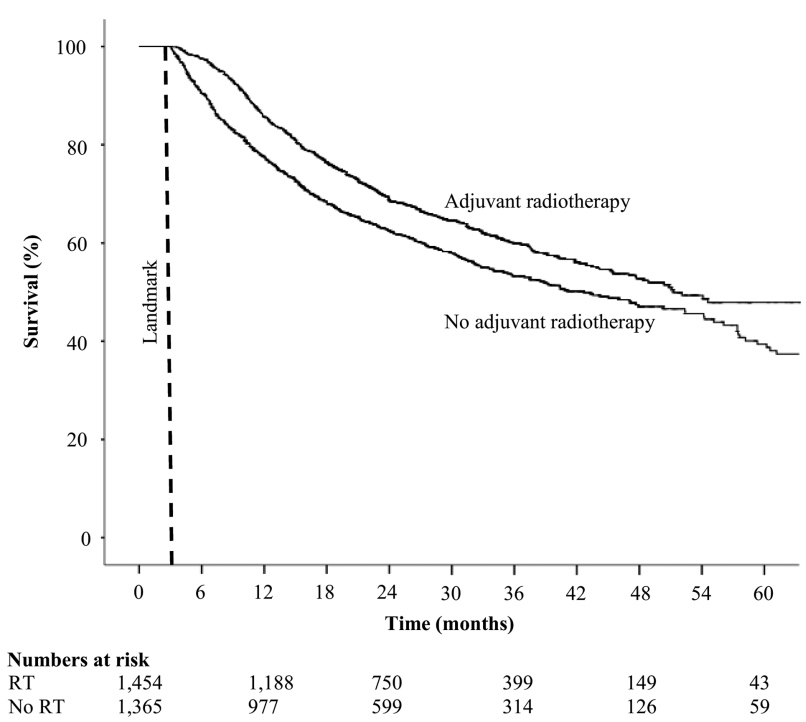

F I G U RE 3 Kaplan-Meier overall survival by landmark analysis of propensity-matched patients alive at 3 months $(P<.001)$ 
TABLE 5 Kaplan-Meier 5-year overall survival stratified by patient and tumor characteristics for propensity-matched patients

\begin{tabular}{llll} 
Variables & No adjuvant RT & Adjuvant RT & Log-rank $\boldsymbol{P}$ value \\
\hline Overall & $37.0 \%( \pm 2.4)$ & $47.7 \%( \pm 2.2)$ & $<.001$ \\
\hline $\begin{array}{l}\text { Age, years } \\
\quad<63\end{array}$ & & & \\
$\quad \geq 63$ & $44.4 \%( \pm 4.3)$ & $53.5 \%( \pm 3.4)$ & .076 \\
\end{tabular}

Charlson/Deyo comorbidity score

$\begin{array}{ll}0 & 38.0 \%( \pm 3.0) \\ 1 & 33.5 \%( \pm 4.1) \\ 2 & 30.2 \%( \pm 6.0)\end{array}$

$\begin{array}{ll}47.8 \%( \pm 2.7) & <.001 \\ 54.6 \%( \pm 3.5) & <.001 \\ 26.5 \%( \pm 7.7) & .635\end{array}$

Extracapsular extension

No

Positive margins

No

N2

T3-4

Yes

Yes

Positive surgical margins

No

Extracapsular extension

No

N2

T3-4

Yes

Yes

$\mathrm{T}$ classification

1

2

3

4

$\mathrm{N}$ classification

$\begin{array}{ll}0 & 47.0 \%( \pm 3.2) \\ 1 & 32.4 \%( \pm 7.2) \\ 2 & 18.9 \%( \pm 4.1) \\ 3 & \ldots\end{array}$

\section{Grade}

1

2

3

Unknown
$41.4 \%( \pm 2.6)$

$44.1 \%( \pm 2.9)$

$29.5 \%( \pm 7.3)$

$44.2 \%( \pm 3.0)$

$24.6 \%( \pm 5.9)$

$15.0 \%( \pm 3.0)$
$50.5 \%( \pm 2.5)$

$51.6 \%( \pm 2.7)$

$30.9 \%( \pm 6.7)$

$51.2 \%( \pm 2.9)$

$43.4 \%( \pm 6.0)$

$32.3 \%( \pm 4.2)$

$49.0 \%( \pm 2.5)$

$51.6 \%( \pm 2.7)$

$30.9 \%( \pm 6.7)$

$51.2 \%$ ( \pm 2.9 )

$33.9 \%( \pm 4.9)$

$39.9 \%( \pm 4.9)$

$50.8 \%( \pm 10.3)$

$45.1 \%( \pm 6.3)$

$43.6 \%( \pm 10.0)$

$47.8 \%( \pm 2.5)$

$58.8 \%( \pm 2.7)$

$43.6 \%( \pm 6.9)$

$25.3 \%( \pm 4.4)$

...
$44.9 \%( \pm 5.2)$

49.7\% ( \pm 2.8 )

$46.0 \%$ ( \pm 5.6$)$

$45.8 \%( \pm 9.0)$
$<.001$

$<.001$

.073

$<.001$

.001

$<.001$

Abbreviation: RT, radiotherapy.

$2013,13.3 \%$ of the entire U.S. population was found to be uninsured for the entire year, and the uninsured rate had decreased to $10.4 \%$ for the calendar year $2014 .^{28}$ The observed increase in adjuvant RT utilization may also be reflective of the NCDB and the possibility of increased capture of RT data by Commission on Cancer institutions over time, whereas the tumor registrar might be more likely to have access to RT records. Linking of hospital and radiation oncology electronic health records and implementation of electronic data capture systems to access population research databases prospectively and better facilitate outcomes reporting have been developed. ${ }^{29,30}$ Further epidemiological 
studies on the utilization of RT for patients with head and neck cancers are warranted.

Our study represents a large comprehensive analysis investigating prognostic factors and the impact of adjuvant RT on patients with resected locoregionally advanced squamous cell carcinoma of the oral cavity. The major strength in our investigation lies in the large patient numbers, which allowed for statistical analyses powered for detection of differences in OS. The NCDB is a highly standardized hospitalbased cancer registry, undergoing a series of quality assurance measures and checks while collecting information on $70 \%$ of patients with newly diagnosed cancer annually. ${ }^{10}$ However, intrinsic limitations to any large database retrospective analysis must also be considered with lack of certain data variables possibly leading to confounding impact of important factors that could not be investigated in our study. Patients from the NCDB are only reported by Commission on Cancer-accredited facilities, thus possibly introducing hospital-selection bias. In addition, the NCDB does not have records on preoperative and postoperative imaging, patient adherence to treatment recommendations, disease recurrence, or any subsequent salvage therapy. There is limited information on some intermediate-risk head and neck cancer features, such as perineural invasion, lymphovascular space invasion, and depth of invasion. Number of involved nodes and nodal stations involved were not specifically analyzed in our study as well. Additionally, risk factors, including tobacco and alcohol use, which are among the strongest disease risk factors for squamous cell carcinoma of the oral cavity, are not specifically recorded. ${ }^{31}$ Thus, we cannot evaluate the extent to which all of these factors may contribute to patient outcome, a limitation that can present a greater risk of unmeasured confounding. Another significant limitation of this study is the short median follow-up. A shorter median follow-up may lead to analyses biased toward patients with shorter follow-up. The interpretation of survival analyses depends on the completeness of patient follow-up, and sufficient follow-up to capture an adequate number of events is necessary to ensure sufficient statistical power for outcomebased data analyses. ${ }^{32,33}$ However, the role of median follow-up in describing the stability and validity of the Kaplan-Meier curve estimates is also debatable, as some argue that Kaplan-Meier curves adjust for variable lengths of follow-up and provide an unbiased estimate of the true population survival curve. ${ }^{34}$ Regardless, median follow-up is a term that continues to be used in outcome-based cancer research, and our short median follow-up warrants the need for future studies with more mature data and longer median follow-up. Finally, the NCDB does not collect cancerspecific survival information, which may produce different results from that of all-cause deaths.

Our findings from this hospital-based retrospective propensity-score matched analysis suggest that receipt of adjuvant RT is associated with significantly improved OS in those with locoregionally advanced squamous cell carcinoma of the oral cavity, and that it should strongly be considered for all clinically suitable patients who have undergone resection for the disease. As techniques for RT delivery continue to be optimized and its utilization increased, further studies are warranted to continue to investigate prospectively which specific subgroups of patients truly benefit from treatment so that disease control and outcomes can be further improved, while minimizing unnecessary treatment toxicity and maintaining quality of life.

\section{ORCID}

Jacob Y. Shin MD iD http://orcid.org/0000-0003-1558-3731

\section{REFERENCES}

[1] Siegel RL, Miller KD, Jemal A. Cancer Statistics, 2017. $C A$ Cancer J Clin. 2017;67:7-30.

[2] Scully C, Bagan J. Oral squamous cell carcinoma overview. Oral Oncol. 2009;45:301-308.

[3] NCCN Guidelines Version 2.2017. Cancer of the Oral Cavity. National Comprehensive Cancer Network. https://www.ncen. org/professionals/physician_gls/pdf/head-and-neck.pdf. Accessed October 10, 2017.

[4] Laskar SG, Chaukar D, Deshpande M, et al. Phase III randomized trial of surgery followed by conventional radiotherapy ( $5 \mathrm{fr} /$ Wk) (Arm A) vs concurrent chemoradiotherapy (Arm B) vs accelerated radiotherapy (6fr/Wk) (Arm C) in locally advanced, stage III and IV, resectable, squamous cell carcinoma of oral cavity- oral cavity adjuvant therapy (OCAT): final results (NCT00193843). J Clin Oncol. 2016;34:(Suppl);abstract 6004.

[5] Robertson AG, Soutar DS, Paul J, et al. Early closure of a randomized trial: surgery and postoperative radiotherapy versus radiotherapy in the management of intra-oral tumours. Clin Oncol (R Coll Radiol). 1998;10:155-160.

[6] Iyer NG, Tan DS, Tan VK, et al. Randomized trial comparing surgery and adjuvant radiotherapy versus concurrent chemoradiotherapy in patients with advanced, nonmetastatic squamous cell carcinoma of the head and neck: 10-year update and subset analysis. Cancer. 2015;121:1599-1607.

[7] Sher DJ, Thotakura V, Balboni TA, et al. Treatment of oral cavity squamous cell carcinoma with adjuvant or definitive intensity-modulated radiation therapy. Int J Radiat Oncol Biol Phys. 2011;81:e215-e222.

[8] Cooper JS, Pajak TF, Forastiere AA, et al. Postoperative concurrent radiotherapy and chemotherapy for high-risk squamous-cell carcinoma of the head and neck. N Engl J Med. 2004;350:1937-1944.

[9] Bernier J, Domenge C, Ozsahin M, et al. Postoperative irradiation with or without concomitant chemotherapy for locally advanced head and neck cancer. N Engl J Med. 2004;350:19451952.

[10] National Cancer Data Base (NCDB).https://www.facs.org/quality-programs/cancer/ncdb. American College of Surgeons. Accessed October 10, 2017. 
[11] Amin MB, Edge S, Greene F, et al. AJCC Cancer Staging Manual, Eighth Edition. Chicago, IL, American Joint Committee on Cancer (AJCC), 2017.

[12] Deyo RA, Cherkin DC, Ciol MA. Adapting a clinical comorbidity index for use with ICD-9-CM administrative databases. J Clin Epidemiol. 1992;45:613-619.

[13] Charlson/Deyo Score. National Cancer Database: Data Dictionary PUF 2014. http://ncdbpuf.facs.org/content/charlsondeyocomorbidity-index. Accessed 10 May 2016.

[14] Austin PC. Some methods of propensity-score matching had superior performance to others: results of an empirical investigation and Monte Carlo simulations. Biom J. 2009;51:171-184.

[15] Liao CT, Chang JT, Wang HM, et al. Analysis of risk factors of predictive local tumor control in oral cavity cancer. Ann Surg Oncol. 2008;15:915-922.

[16] Parsons JT, Mendenhall WM, Stringer SP, et al. An analysis of factors influencing the outcome of postoperative irradiation for squamous cell carcinoma of the oral cavity. Int J Radiat Oncol Biol Phys. 1997;39:137-148.

[17] Hinerman RW, Mendenhall WM, Morris CG, et al. Postoperative irradiation for squamous cell carcinoma of the oral cavity: 35-year experience. Head Neck. 2004;26:984-994.

[18] Laramore GE, Scott CB, Schuller DE, et al. Is a surgical resection leaving positive margins of benefit to the patient with locally advanced squamous cell carcinoma of the head and neck: a comparative study using the intergroup study 0034 and the Radiation Therapy Oncology Group head and neck database. Int J Radiat Oncol Biol Phys. 1993;27:1011-1016.

[19] Rosenthal DI, Mohamed ASR, Garden AS, et al. Final report of a prospective randomized trial to evaluate the dose-response relationship for postoperative radiation therapy and pathologic risk groups in patients with head and neck cancer. Int J Radiat Oncol Biol Phys. 2017;98:1002-1011.

[20] Maxwell JH, Ferris RL, Gooding W, et al. Extracapsular spread in head and neck carcinoma: impact of site and human papillomavirus status. Cancer. 2013;119:3302-3308.

[21] Thomas B, Stedman M, Davies L. Grade as a prognostic factor in oral squamous cell carcinoma: a population-based analysis of the data. Laryngoscope. 2014;124:688-694.

[22] Ang KK, Trotti A, Brown BW, et al. Randomized trial addressing risk features and time factors of surgery plus radiotherapy in advanced head-and-neck cancer. Int J Radiat Oncol Biol Phys. 2001;51:571-578

[23] Sogaard M, Thomsen RW, Bossen KS, et al. The impact of comorbidity on cancer survival: a review. Clin Epidemiol. 2013; 5:3-29.

[24] Lee NY, de Arruda FF, Puri DR, et al. A comparison of intensity-modulated radiation therapy and concomitant boost radiotherapy in the setting of concurrent chemotherapy for locally advanced oropharyngeal carcinoma. Int J Radiat Oncol Biol Phys. 2006;66:966-974.

[25] Guadagnolo BA, Liu CC, Cormier JN, et al. Evaluation of trends in the use of intensity-modulated radiotherapy for head and neck cancer from 2000 through 2005: socioeconomic disparity and geographic variation in a large population-based cohort. Cancer. 2010;116:3505-3512.

[26] Inverso G, Mahal BA, Aizer AA, et al. Health insurance affects head and neck cancer treatment patterns and outcomes. J Oral Maxillofac Surg. 2016;74:1241-1247.

[27] Affordable Care Act, About the Law. U.S. Department of Health \& Human Services. http://www.hhs.gov/healthcare/about-thelaw/. Accessed October 10, 2017.

[28] Smith JC, Medalia C. Health Insurance Coverage in the United States: 2014. Washington, DC: U.S. Census Bureau, U.S. Government Printing Office.

[29] Russo GA. When electronic health records (EHRs) talk, everyone can win: our experience creating a software link between hospital and radiation oncology EHRs. Int $J$ Radiat Oncol Biol Phys. 2016;94:206-207.

[30] Pan HY, Shaitelman SF, Perkins GH, et al. Implementing a realtime electronic data capture system to improve clinical documentation in radiation oncology. J Am Coll Radiol. 2016;13:401407.

[31] What Are the Risk Factors for Oral Cavity and Oropharyngeal Cancers? American Cancer Society. Last revised: August 8, 2016. https://www.cancer.org/cancer/oral-cavity-and-oropharyngeal-cancer/causes-risks-prevention/risk-factors.html. Accessed March 16, 2017

[32] Altman DG, De Stavola BL, Love SB, et al. Review of survival analyses published in cancer journals. Br J Cancer. 1995;72: 511-518

[33] Clark TG, Bradburn MJ, Love SB, et al. Survival analysis part I: basic concepts and first analyses. Br J Cancer. 2003;89:232238 .

[34] Shuster JJ. Median follow-up in clinical trials. J Clin Oncol. 1991;9:191-192.

How to cite this article: Shin JY, Yoon JK, Shin AK, Diaz AZ. Locoregionally advanced oral cavity cancer: A propensity-score matched analysis on overall survival with emphasis on the impact of adjuvant radiotherapy. Head \& Neck. 2018;40:1934-1946. https:// doi.org/10.1002/hed.25185 